Volume 8. No. 7, July 2020

International Journal of Emerging Trends in Engineering Research

Available Online at http://www.warse.org/IJETER/static/pdf/file/ijeter132872020.pdf

https://doi.org/10.30534/ijeter/2020/132872020

\title{
The Technique of Research on the Development of Radar Methods of Small Air Objects Detection
}

\author{
Hennadii Khudov', Serhii Yarosh', Vitalii Savran ${ }^{3}$, \\ Andrii Zvonko ${ }^{4}$, Andrii Shcherba ${ }^{5}$, Pavlo Arkushenko ${ }^{6}$ \\ ${ }^{1}$ Department of Radar Troops Tactic, Ivan Kozhedub Kharkiv National Air Force University, Kharkiv, Ukraine, \\ 2345kh_hg@ukr.net \\ ${ }^{2}$ Department of Anti-aircraft Missiles Tactic, Ivan Kozhedub Kharkiv National Air Force University, Kharkiv, \\ Ukraine, syarosh@ukr.net \\ ${ }^{3}$ Research center, Military Institute of Taras Shevchenko Kyiv National University, Kyiv, Ukraine, \\ vitaliy-savran@ukr.net \\ ${ }^{4}$ Department of Rocket Artillery Armament, Hetman Petro Sahaidachnyi National Army Academy, Lviv, \\ Ukraine, zvonko2008@ukr.net \\ ${ }^{5}$ Department of Artillery Facility Complexes and Devices, Hetman Petro Sahaidachnyi National Army Academy, \\ Lviv, Ukraine, 2345kh_hg@ukr.net \\ ${ }^{6}$ Department of Metrological Assessments and Examinations, State Research Institute forTesting and \\ Certification of Arms and Military Equipment, Chernigiv, Ukraine, ap11981@ukr.net
}

\begin{abstract}
The paper proposes the technique of research on the development of radar methods of small air objects detection. The technique includes: using of calculation methods for determining the characteristics of secondary radiation of small air objects in single-position and multi-position reception, using of multiposition radar methods, the methods of mathematical modeling of air object detection processes in radar systems.

It is established that using of methods of mathematical (simulation) modeling will allow to conduct statistical experiments, calculate the characteristics of detection of small air objects and compare the effectiveness of different radar methods. Simulation of the process of detecting air objects should be performed using the Monte Carlo method, probability theory and mathematical statistics.
\end{abstract}

Key words : the technique, radar, small air objects, detection, multiposition, mathematic modeling.

\section{INTRODUCTION}

The main trends in the development of air objects at the present stage are due to the introduction of modern technologies and are as follows [1]-[17]:

- the improvement of flight and tactical characteristics;

- the reduction of radar visibility;

- the improvement of on-board electronic equipment and fire means;

- the introduction of unmanned control and the latest information technologies.
Promising air objects tend to be suitable both for using in large-scale warfare and for use in local armed conflicts, hybrid and cold war [1], [8]-[10], [13]-[17].

Features of radar detection of small air objects are [2], [5]-[6], [8], [18]-[26]:

- the short detection range;

- finding air objects in the area of illumination from local objects, which requires the inclusion of equipment for protection against passive interference, which, in turn, reduces the detection range;

- the small effective scattering surface.

It is known that to increase the efficiency of detection of small air objects, a number of organizational and technical measures are used [18]-[26]:

- the compaction of the location of the radar in dangerous directions (creation of detection bands of low-altitude and small-sized objects);

- using of radars of all frequency bands;

- using of radars with the best capabilities (greatest energy potential) and more.

\subsection{Problem analysis}

Information on the monostatic and bistatic effective scattering surface of objects can be obtained by the following methods [18]-[26]:

- measurement of the effective scattering surface on special radar landfills, in the field or in radio frequency anechoic chambers using real objects or their large-scale copies;

- analytical calculations;

- mathematical modeling using numerous methods.

Known methods of increasing the detection range of small air objects used in radar are[18]-[26]:

- increasing the energy potential of the radar; 
- improving the quality of radar signal processing;

- change the range of operating frequencies of the radar;

- using of multi-position radar systems;

- using of sounding signals that are consistent with the shape of the air object.

The increase in energy potential and the use of sounding signals, which are consistent with the shape of the air object, is associated with the complexity of transmitting devices and increasing power consumption or dimensions of the radar.

Mathematical modeling is the most powerful and universal method of research and evaluation of the effectiveness of systems whose behavior depends on the influence of random factors. Mathematical modeling is based on a statistical experiment (Monte Carlo method).

In the study of radar systems, there are often cases when an accurate analytical solution of problems is impossible, and the evaluation of characteristics using field tests causes significant difficulties. In addition, it is not always possible to create all the necessary conditions for field tests. Therefore, for the study of radar systems it is advisable to use mathematical (simulation) models. A mathematical (simulation) model is a conditional mathematical description that is in some relation to the object under study and is able to replace it during research, to give information about it. Mathematical modeling is to conduct experiments with a machine program that simulates the operation of the system under random influences, followed by statistical processing of the simulation results [18]-[26].

The method of mathematical (simulation) modeling allows you to build models that describe the processes as they would actually take place. Such a model can be "lost" in time for both one test and a given set of them. The results will be determined by the random nature of the processes. According to these data, you can get fairly stable statistics. Different methods are used to mathematically describe the input effects and internal phase transformations. To solve the problems of modeling the processing and detection of radar signals is convenient to use the method of complex bypass[20]-[22].

The ultimate goal of mathematical (simulation) modeling is to help make an informed decision about the quality of the modeling object in accordance with the theory of decision making. The result of the simulation is evaluated using an efficiency indicator. Comparison of modeling results of different strategies is carried out on the basis of efficiency. The indicator and the criterion of efficiency together reflect the purpose of modeling and the way to achieve it. Therefore, when developing a mathematical (simulation) model, it is necessary to clearly define the indicator and criterion of efficiency, which reflect the system (set) of advantages when making comparisons and decision-making.
The purpose of the article is to develop the technique of research on the development of radar methods of small air objects detection.

\section{MAIN MATERIAL}

The technique of research on the development of radar methods of small air objects detection includes: using of calculation methods for determining the characteristics of secondary radiation of small air objects in single-position and multi-position reception, using of multiposition radar methods, the methods of mathematical modeling of air object detection processes in radar systems.

\subsection{Using of calculation methods for determining the characteristics of secondary radiation of small air objects in single-position and multi-position reception}

The development of methods to increase the efficiency of detection of small air objects in survey radars due to the integrated use of the properties of bistatic and monostatic effective scattering surface is based on the use of electrodynamic scattering theory and principles of applied electrodynamics.

It is known that the value of the effective scattering surface depends on the geometry and electrical properties of the material of the air object, the ratio of its geometric dimensions and wavelength, the angle of irradiation, the polarization of electromagnetic waves irradiating the object. When electromagnetic waves are scattered on an air object of complex shape, a change in the polarization of the incident wave can be observed, due to which components appear in the reflected signal, which are orthogonal to the probing signal in terms of polarization. Therefore, the numerical value of the effective scattering surface is valid only for a certain type of object, the combination of polarization, angle and frequency for which it was determined. In practice, the value of the effective scattering surface can vary within a wide range of 20-30 dB or more with a relatively small change in any of these parameters [26]. Changing the parameters of the radar can affect the value of the effective scattering surface, which determines the main ways to increase the detection range of small air objects [126]. The scattering properties of an air object, in the General case, depend on the direction of irradiation and reception. The bistatically effective scattering surface may not coincide with the monostatically effective scattering surface of the same air object. When changing the orientation of an air object in space, the value of the monostatic effective scattering surface is a function of two angles (azimuth and angle), and the value of the bistatic effective scattering surface is a function of four angles (two azimuths and two angles). In real air objects during the flight, the fluctuations of the angle of the transmitting and receiving positions at each time point are a random variable. Therefore, the value of the effective scattering surface relative to the receiving 
position will also vary randomly. We can assume that the form of this law for the monostatic and bistatic effective scattering surface will be different. This allows us to hypothesize a possible general increase in the value and change in the nature of fluctuations of the effective scattering surface of the air object with the integrated use of the properties of its bistatic and monostatic effective scattering surface.

Currently, the using of calculation methods for mathematical modeling of radar characteristics of aircraft, which take into account the irregularities of their surfaces, the presence of radio-absorbing coatings and other factors that complicate the use of analytical methods. Representation of air objects that have a complex shape, facet model and the use of mathematical modeling to study their effective scattering surface provides an opportunity to take into account the features of scattering of electromagnetic waves by an air object at a spaced reception.

Known methods for calculating the effective scattering surface are based on the representation of the scattered field by the Chu-Stregton integral or its simplifications. The exact solution of the scattering problem using the Chu-Stretton integral is possible only with a known distribution of electric and magnetic fields on the surface of the body, which is quite complex. In practice, approximate theories are used. The simplest approximate theory is based on the use of geometric optics, but it does not take into account the effect of polarization on the interference of waves during scattering, which causes fluctuations in the effective scattering surface, which are characteristic of most air objects. The second approximate theory is based on the use of physical optics, which takes into account the peculiarities of interference, but does not take into account the effects of depolarization in the scattering of radio waves. The third approximate theory is based on the use of the geometric theory of diffraction, which is the development of the theory of geometric optics and taking into account the effects of diffraction. The peculiarity of this theory is that the concept of scattering centers is introduced for sections of a geometric body (except for sections near geometric gaps) and an additional phase factor is introduced, which is proportional to the distance in wavelength from the scattering center to the radar. This makes it possible to take into account the rapid fluctuations of the effective scattering surface from the angle of the air object and to conduct studies of changes in the effective scattering surface from the value of the bistatic angle at spaced reception.

To study the properties of the effective scattering surface of small air objects, the methods of stationary phase and physical optics are used, which will be developed in the monograph on solving radar problems for cases of spaced reception and nonstationary sounding. When calculating the bistatic effective scattering surface, it is taken into account that the principle of reciprocity is not fulfilled in bistatic reception. Using of the methods proposed provides the possibility of calculating the effective scattering surface with arbitrary orientation of the plane of separation of light and shadow relative to the direction of irradiation, in the presence of fracture of the surface and radio-absorbing coatings.

The distribution of the values of the amplitude multiplier of the signal reflected from the air object is estimated on the assumption that the angle of the air object at each time point relative to the radar antennas is a random variable. When the size of the air object is greater than the wavelength of the phase signals, which are reflected by different parts of the air object, at the input of the receiver can have arbitrary values. Therefore, the sum of all signals will be a random variable. In this case, minor movements of the air object around its center of mass lead to significant changes in the distances relative to the wavelength, which causes sharp changes in the phase of the signals reflected from different parts of the air object. This phenomenon is the basis for modeling the fluctuations of the amplitude and phase of the total signal at the input of the receiver. The distribution law of the amplitude factor is determined from histograms of the calculated values of the instantaneous effective scattering surface using the Kolmogorov-Smirnov agreement criterion.

Thus, the using of mathematical modeling methods based on the facet model of the object, separate estimation of the contributions of the smooth surface and edge, provide the calculation of monostatic and bistatic effective scattering surface of the air object, which have a complex shape and are made of conductive, dielectric or composite materials. Testing the hypothesis of a possible general increase in the value and change in the nature of fluctuations of the effective scattering surface of an air object with the integrated use of the properties of its bistatic and monostatic effective scattering surface should be carried out using mathematical modeling, electromagnetic field theory, approximations of geometric and physical optics. geometric theory of diffraction.

\subsection{Using of multiposition radar methods}

Promising ways to improve the quality of detection of small air objects are the use of multi-position radar methods and improve the quality of radar signal processing. The simplest version of the multi-position radar radar system, which can improve the detection of small air objects, is a bistatic radar. When analyzing the differences between a bistatic radar and a combined radar to detect small air objects, it is advisable to use a bistatic angle (the angle between the directions from the target to the transmitting and receiving position) as the main parameter. The magnitude of the bistatic angle determines the differences between the bistatic and monostatic effective scattering surface of the air object. The assessment of the range of the radar for the detection of air objects is based on the assessment of the signal / 
noise ratio, which is provided at the output of the echo signal processing system. In multi-position radar systems, the signal-to-noise ratio at the output of the signal processing system depends on many factors, in particular:

- technical characteristics of receiving and transmitting positions;

- changes in antenna gain during scanning;

- system geometry (number and spatial arrangement of positions);

- modes of operation and method of combining information.

For constructing the area of action of a multi-position radar system, the condition of a constant effective scattering surface of an air object is used. At the same time, the difference in the values of the effective scattering surface, which are due to the change in the angle and bistatic angle of the air object during its movement, are significant. Therefore, when estimating the range of a multi-position radar system for the detection of small air objects, it is advisable to use the features of changing the effective scattering surface for certain types of air objects along with the conditionality of the effective scattering surface.

If the multi-position radar has one transmitting and several receiving positions or one receiving and several transmitting positions, it is possible to implement the following known types of multi-position radar system and methods of combining information:

- spatial-coherent radar with integration of information from spaced positions at the level of radio signals;

- spatially incoherent radar or with short-term spatial coherence and integration at the level of video signals;

- spatially incoherent radar or with short-term spatial coherence and integration at the level of unit measurements;

- spatially incoherent radars with a combination of trajectories.

The method of combining information used in a multi-position radar system determines the system's potential for detecting airborne objects and depends on the degree of coherence of the echoes received at the receiving positions.

When receiving spatially coherent non-fluctuating echoes or signals that have friendly amplitude fluctuations in different positions, it is possible to implement spatially coherent processing and combining information from different positions at the level of radio signals. When receiving spatially incoherent signals, or signals with uncorrelated amplitude fluctuations in different positions, other ways of combining information are implemented.

Therefore, when conducting a study of the possibility of improving the quality of detection of small air objects using multi-position radar methods, it is necessary:

- determine restrictions on the location of receiving and transmitting positions;
- determine restrictions on the relative distance between the air object and the positions of the multi-position radar system;

- determine the restrictions on the type of air objects and the peculiarities of the construction of its body;

- to determine the nature of fluctuations and the degree of spatial coherence of echo signals reflected from the air object and received in spatially spaced receiving positions at different values of the bistatic angle;

- to determine the possible type of multi-position radar system, the method of combining information and solving the problem of detecting an air object.

The problem of detecting an air object is solved after the coordinated processing of echo signals received in the receiving positions, using the known criterion of the likelihood ratio (1):

$$
\begin{aligned}
& \Lambda=\exp \left\{\operatorname{Re} \int_{-\frac{T}{2}-\frac{T}{2}}^{\frac{T}{2}} \int^{\frac{T}{2}} S^{*}\left(t_{1}, \Theta\right) R\left(t_{1}, t_{2}\right) X\left(t_{2}\right) d_{1} d_{t_{2}},\right. \\
& \left.-0,5 \int_{-\frac{T}{2}-\frac{T}{2}}^{\frac{T}{2}} \int_{-\frac{T}{2}}^{\frac{T}{2}} S^{*}\left(t_{1}, \Theta\right) R\left(t_{1}, t_{2}\right) S\left(t_{2}, \Theta\right) d t_{1} d_{2}\right\}
\end{aligned}
$$

where $\Lambda$ is the general ratio of the plausibility of regular signals in the Gaussian distribution of interference and noise in the $\mathrm{m}$-channel receiving system; $\mathrm{X}(\mathrm{t})=\left(\mathrm{x}_{1}(\mathrm{t}), \ldots\right.$, $\left.\mathrm{x}_{\mathrm{m}}(\mathrm{t})\right)$ is the complex form of recording the vector of implementations to accept in the receiving channels (positions); $\mathrm{S}^{*}\left(\mathrm{t}_{1}, \Theta\right)=\left(\mathrm{S}_{1}^{*}\left(\mathrm{t}_{1}, \Theta\right), \ldots, \mathrm{S}_{\mathrm{m}}^{*}\left(\mathrm{t}_{1}, \Theta\right)\right)$ is the complex form of recording the vector of useful signals expected in the receiving channels (positions); $\Theta$ is the vector of signal parameters; $T$ is the interval of reception of implementations in the receiving channels (positions); $\mathrm{R}\left(\mathrm{t}_{1}, \mathrm{t}_{2}\right)$ is the matrix dimension, which is obtained from the solution of the integral-matrix equation (2):

$$
\int_{-\frac{T}{2}}^{\frac{T}{2}} \mathrm{~B}\left(\mathrm{t}_{1}, \mathrm{t}\right) \mathrm{R}\left(\mathrm{t}, \mathrm{t}_{2}\right) \mathrm{dt}=\mathrm{I} \delta\left(\mathrm{t}_{1}-\mathrm{t}_{2}\right) \quad-\frac{\mathrm{T}}{2} \leq\left(\mathrm{t}_{1}, \mathrm{t}_{2}\right) \leq \frac{\mathrm{T}}{2},
$$

where I is a single matrix of the $\mathrm{m}$-th order; $\delta(\mathrm{t})$ is the delta function; $\mathrm{B}\left(\mathrm{t}_{1}, \mathrm{t}_{2}\right)$ is the spatio-temporal correlation matrix of the sum of external interference and intrinsic noise dimension.

To compare the quality of detection of an air object using optimal and suboptimal detection algorithms, detection characteristics are used, which are calculated or measured with a fixed probability of false alarm.

A potential assessment of the quality of detection of an air object is an assessment that is determined using the optimal algorithm for detecting an air object against the background of interference with a deterministic correlation matrix $\mathrm{B}\left(\mathrm{t}_{1}, \mathrm{t}_{2}\right)$, in particular, against the background noise of the receiving device. 
The specification of implementation processing algorithms $\mathrm{X}(\mathrm{t})$, which in the general case is determined by expression (1), depends on the type of matrices of external interference and intrinsic noise $\mathrm{B}\left(\mathrm{t}_{1}, \mathrm{t}_{2}\right)$ in certain operating conditions of multi-position (multi-channel) radar system.

A feature of the joint processing (combining) of signals received by spatially spaced receiving channels is the need to take into account different values of Doppler frequency shift, delays, phase shifts and signal-to-noise ratios in different receiving channels.

Thus, the use of plausibility criteria, the theory of multi-channel signal processing and multi-position radar will provide an opportunity to develop a method to improve the detection of small air objects with integrated use of the properties of bistatic and monostatic effective scattering surface. It is advisable to assess the quality of detection of air objects by comparing the characteristics of detection, which are provided by using different methods of radar and signal processing. The study of detection characteristics should be carried out using mathematical modeling methods, taking into account the features of bistatic and monostatic effective scattering surface for air objects of complex shape, using the theory of statistical radio engineering and multi-position radar.

\subsection{The methods of mathematical modeling of air object detection processes in radar systems}

Using of computer modeling technology to create a mathematical (simulation) model involves the following steps:

- determining the purpose of modeling;

- the development of a conceptual model;

- the formalization of the model;

- the software implementation of the model;

- planning of experiments;

- the analysis and interpretation of modeling results.

To solve the problems of the study it is necessary to compare the effectiveness of detecting small software, which is provided by using different methods under different conditions and input effects. As an indicator of efficiency when comparing different methods, the value of the required signal-to-noise threshold ratio is used, which provides detection of small software with specified quality indicators. As a criterion of efficiency the minimization of the value of the required threshold signal / noise ratio is used.

To achieve this goal, it is necessary to form a set of experimental data based on the results of statistical tests (Monte Carlo method), which will provide an assessment of the characteristics of detection and determination of quality indicators (signal-to-noise threshold).

The essence of the method of statistical tests (Monte Carlo method) is that the test result depends on the values of a random variable, which is distributed according to a certain law. Therefore, the result of each individual test is random. Statistical data obtained, processed and presented in the form of appropriate estimates of certain parameters (characteristics). The theoretical basis of the method of statistical tests are the boundary theorems of probability theory (Chebyshev's, Bernoulli's, Poisson's theorem), which guarantee high quality statistical estimates for a large number of tests [128-130].

To conduct a model experiment, it is necessary to determine:

- the required duration of the run to achieve stationary conditions;

- how to obtain statistically independent observations;

- the number of observations required to achieve a certain accuracy.

For researches of processes of detection of small-sized software at one-position and multi-position reception the simulation model should consist of the following structural components (figure 1):

- the block for generating source data for modeling with a graphical interface;

- the block for simulating the parameters of the spatial configuration of the system;

- the block for simulating a single-position effective scattering surface of the air object relative to the combined transceiver position;

- the block for simulating the bistatic effective scattering surface of the air object relative to the spaced receiving and transmitting positions;

- the block for simulating the processing of echo signals and the detector;

- the block of statistics collection;

- the block of estimation of statistical characteristics;

- the block analysis of results with a graphical interface.

The block of formation of initial data for modeling provides process of simulation modeling by the decision of the following basic problems:

- the determination of the initial coordinates of air objects;

- the determination of coordinates of positions;

- the choice of method (algorithm) for combining radar information

The block for simulating the parameters of the spatial configuration of the system provides the calculation of the distance and angles of the air object relative to the receiving and transmitting positions. The calculated parameters are used to calculate the values of the corresponding effective scattering surfaces of air objects.

The simulation block of single-position and bistatic scattering surfaces of county objects provide calculation of the value of the corresponding effective scattering surface of the air object relative to a certain receiving position taking into account the angle of the air object, bistatic angle and slight movements of the air object around its center of mass. The calculated values are used 
to calculate the amplitudes of the echo signals received at the respective receiving positions.

The block for simulating the processing of echo signals and the detector provides simulation of the own noise of the receiving devices and the calculation of the amplitude of the echo signals received in the receiving positions, taking into account:

- parameters of the corresponding transmitting devices; - the distance between the transmitting device, the air object and the receiving device;

- values the effective surface of the object, which are calculated taking into account the angles and bistatic angles of the air object.

The block of statistics collection provides data accumulation during statistical runs of the model for further calculation of statistical characteristics.

The block for estimating statistical characteristics provides the calculation of statistical characteristics of the detection of the air object using different detection methods according to the data that were accumulated during the statistical runs of the model.

The estimation of the probability of occurrence of the event (correct detection or false alarm) based on the results of statistical runs of the model is determined by expression (3):

$$
\hat{\mathrm{p}}=\frac{\mathrm{k}_{\mathrm{A}}}{\mathrm{N}}
$$

where $\hat{p}$ is the estimate the probability of event $A ; k_{A}$ is the number of runs of the model where event $\mathrm{A}$ was observed; $\mathrm{N}$ is the total number of runs of the simulation model.

The number of required runs $\mathrm{N}$ is determined from condition (4):

$$
\mathrm{P}\{|\hat{\mathrm{p}}-\mathrm{p}| \leq \mathrm{d}\}=1-\alpha
$$

where $\mathrm{P}$ is the probability of event $\mathrm{A} ; \mathrm{d}$ is the maximum deviation of the estimate $\hat{\mathrm{p}}$ from the probability $\mathrm{P} ;(1-\alpha)$ is the probability that the estimate will deviate from the value by no more than the value.

When the condition $\mathrm{Np}(1-\mathrm{p}) \geq 25$ is met, the required number of runs to ensure the fulfillment of condition (4) is determined by expression (5) [131]:

$$
\mathrm{N}=\frac{\mathrm{Z}_{\alpha / 2}^{2}}{4 \mathrm{~d}^{2}},
$$

where $Z_{\alpha / 2}$ is the standard normal statistics for a certain probability.

The condition of validity, non-bias and asymptotic normality is checked by convincing the convergence of estimates with increasing number of runs.

For radar systems, the value of the probability of false alarm is in the range $p=10^{-5}-10^{-7}$. To increase the accuracy of measuring small probability values with restrictions on the number of statistical experiments, methods of increasing the accuracy of statistical estimates are used, for example, a method of forcing random effects, which is based on replacing the corresponding probability distributions and then recalculating to baseline conditions. But to apply such method in the considered model is inexpedient as in signal processing system adaptive detection algorithms which can be sensitive to changes of characteristics of distributions of probabilities can be applied.

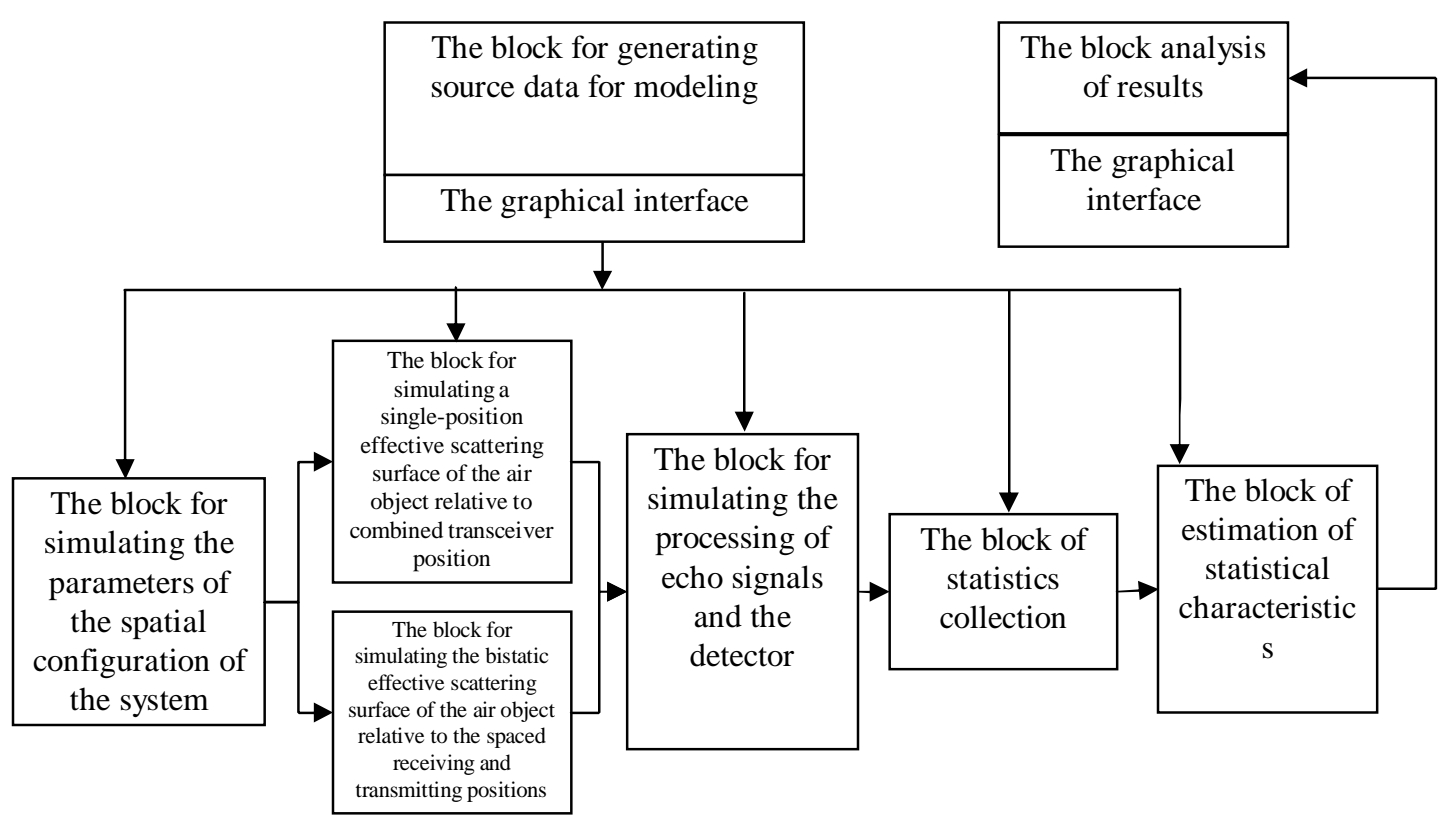

Figure 1: Block diagram of a mathematical model of the process of detecting air objects in multi-position radar systems 
In this case, the recalculation of probabilities measured for one parameter by the probability distributions to other parameters may be incorrect. Therefore, to measure the probability of false alarm in the model, it is advisable to use expression (6):

$$
\hat{\mathrm{p}}=\frac{\mathrm{k}_{\mathrm{nF}}}{\mathrm{N} \cdot \mathrm{M}},
$$

where $\mathrm{k}_{\mathrm{nF}}$ is the number of false alarm events observed during statistical runs; $M$ is the number of uncorrelated separation elements analyzed in each statistical run.

The block of analysis of results provides visual representation of results of calculation of characteristics of detection of small-sized air objects at use of various methods for an estimation of their efficiency. Thus, using of methods of mathematical (simulation) modeling will provide the ability to conduct statistical experiments, calculate the characteristics of the detection of small air objects and compare the effectiveness of different radar methods. Simulation modeling of the process of detecting air objects should be performed using the Monte Carlo method, probability theory and mathematical statistics.

\section{CONCLUSION}

The paper proposes the technique of research on the development of radar methods of small air objects detection. The technique includes: using of calculation methods for determining the characteristics of secondary radiation of small air objects in single-position and multi-position reception, using of multiposition radar methods, the methods of mathematical modeling of air object detection processes in radar systems.

It is established that using of methods of mathematical (simulation) modeling will allow to conduct statistical experiments, calculate the characteristics of detection of small air objects and compare the effectiveness of different radar methods. Simulation of the process of detecting air objects should be performed using the Monte Carlo method, probability theory and mathematical statistics.

\section{REFERENCES}

1. M. Banasik, Armed Forces As The Russian Federation's Strategic Tool, Journal on Baltic Security, № 5 (2), 2019, pp. 1-12.

DOI: https://doi.org/10.2478/jobs-2019-0008.

2. H. Khudov, V. Lishchenko, B. Lanetskii, V. Lukianchuk, S. Stetsiv, and I. Kravchenko The coherent signals processing method in the multiradar system of the same type two-coordinate surveillance radars with mechanical azimuthal rotation, International Journal of Emerging Trends in Engineering Research, Vol. 8. № 6, 2020, pp. 2624-2630.

DOI: https://doi.org/10.30534/ijeter/2020/66862020.
3. H. Khudov, V. Lishchenko, H. Hyshko, Y. Polonskyi, I. Khizhnyak, B. Riabukha, The MIMO Surveillance Radars System with High Accuracy Finding 2D Coordinates, International Journal of Emerging Trends in Engineering Research, Vol. 8. № 5, 2020, pp. 2026-2030.

DOI: https://doi.org/10.30534/ijeter/2020/91852020.

4. H. Khudov, S. Glukhov, O. Maistrenko, A. Fedorov, A. Andriienko, O. Koplik, The Method of ADS-B Receiver Systems Synchronization Using MLAT Technologies in the Course of Radar Control of Air Environment, International Journal of Emerging Trends in Engineering Research, Vol. 8. № 5, 2020, pp. 1946-1951.

DOI: https://doi.org/10.30534/ijeter/2020/78852020.

5. V. Lishchenko, T. Kalimulin, I. Khizhnyak, and H. Khudov, The method of the organization coordinated work for air surveillance in MIMO radar, Paper presented at the 2018 International Conference on Information and Telecommunication Technologies and Radio Electronics, UkrMiCo, 2018 - Proceeding. DOI:https://doi.org/doi:10.1109/UkrMiCo43733.2018. 9047560.

6. O. Barabash, N. Dakhno, H. Shevchenko, and T. Majsak Dynamic Models of Decision Support Systems for Controlling UAV by Two-Step Variational-Gradient Method, Proceedings of 2017 IEEE 4th International Conference "Actual Problems of Unmanned Aerial Vehicles Developments (APUAVD)", October 17-19, 2017, Kyiv, Ukraine. pp. 108-111. DOI:https://doi.org/10.1109/APUAVD.2017.8308787

7. O. Mashkov, V. Sobchuk, O. Barabash, N. Dakhno, H. Shevchenko, and T. Maisak Improvement of variational-gradient method in dynamical systems of automated control for integro-differential models. Mathematical Modeling and Computing, 2019, Vol. 6, № 2, pp. 344-357.

DOI: https://doi.org/10.23939/mmc2019.02.344

8. C. Pascarelli, M. Marra, G. Avanzini, and A. Corallo Environment for Planning Unmanned Aerial Vehicles Operations Aerospace, 2019, 6 (5), 51.

DOI: https://doi.org/10.3390/aerospace6050051

9. H. Khudov, I. Ruban, O. Makoveichuk, H. Pevtsov, V. Khudov, I. Khizhnyak, S. Fryz, V. Podlipaiev, Y. Polonskyi, and R. Khudov. Development of methods for determining the contours of objects for a complex structured color image based on the ant colony optimization algorithm, Eureka: Physics and Engineering, № 1, 2020, pp. 34-47. DOI: https://doi.org/10.21303/2461-4262.2020.001108.

10. I. Ruban, H. Khudov, V. Khudov, I. Khizhnyak, and O. Makoveichuk. Segmentation of the images obtained from onboard optoelectronic surveillance systems by the evolutionary method, Eastern-European Journal of Enterprise Technologies, № 5/9 (89), 2017, pp. 49-57. DOI: https://doi.org/10.15587/1729-4061.2017.109904. 
11. H. Khudov, A. Fedorov, D. Holovniak, and G. Misiyuk. Improving the Efficiency of Radar Control of Airspace with the Multilateration System Use, in Intern. Scient.-Pract. Conf. Problems of Infocommunications. Science and Technology (PIC S\&T), 2018, pp. 680-684. DOI: https://doi.org/10.1109/infocommst.2018.8632141.

12. I. Ruban, H. Khudov, O. Makoveichuk, I. Khizhnyak, N. Lukova-Chuiko, G. Pevtsov, Y. Sheviakov, I. Yuzova, Y. Drob, and O. Tytarenko, Method for determining elements of urban infrastructure objects based on the results from air monitoring, Eastern-European Journal of Enterprise Technologies, № 4/9 (100), 2019, pp. 52-61.

DOI: https://doi.org/10.15587/1729-4061.2019.174576.

13. H. Khudov, S. Glukhov, V. Podlipaiev, V. Pavlii, I. Khizhnyak, and I. Yuzova The Multiscale Image Processing Method from On-board Earth Remote Sensing Systems Based on the Artificial Bee Colony Algorithm, International Journal of Advanced Trends in Computer Science and Engineering, Vol. 9. № 3, 2020, pp. 2557-2562.

DOI: https://doi.org/10.30534/ijatcse/2020/11932020.

14. I. Ruban, H. Khudov, O. Makoveichuk, I. Khizhnyak, V. Khudov, V. Podlipaiev, V. Shumeiko, O. Atrasevych, A. Nikitin, and R. Khudov. Segmentation of opticalelectronic images from on-board systems of remote sensing of the Earth by the artificial bee colony method, Eastern-European Journal of Enterprise Technologies, № 2/9 (98), 2019, pp. 37-45.

DOI: https://doi.org/10.15587/1729-4061.2019.161860.

15. I. Ruban, H. Khudov, O. Makoveichuk, M. Chomik, V. Khudov, I. Khizhnyak, V. Podlipaiev, Y. Sheviakov, O. Baranik, and A. Irkha. Construction of methods for determining the contours of objects on tonal aerospace images based on the ant algorithms, Eastern-European Journal of Enterprise Technologies, № 5/9 (101), 2019, pp. 25-34.

https://doi.org/10.15587/1729-4061.2019.177817.

16. G. V. Khudov, Features of optimization of two-alternative decisions by joint search and detection of objects. Problemy Upravleniya I Informatiki (Avtomatika), 2003, № 5, pp. 51-59.

17. I. Ruban, and H. Khudov, Advances in Spatio-Temporal Segmentation of Visual Data, Chapter 2. Swarm Method of Image Segmentation. Series Studies in Computational Intelligence (SCI), Vol. 876. - Publisher Springer, Cham, $2020 . \quad-\quad$ P. 53-99. DOI https://doi.org/10.1007/978-3-030-35480-0

18. V. Lishchenko, H. Khudov, V. Tiutiunnyk, V._Kuprii, F._Zots, and G._Misiyuk. The Method of Increasing the Detection Range of Unmanned Aerial Vehicles In Multiradar Systems Based on Surveillance Radars, in 2019 IEEE 39th International Conference on Electronics and Nanotechnology (ELNANO), 2019. P. 559-562. DOI: https://doi.org/10.1109/ELNANO.2019.8783263.

19. H. Khudov, A. Zvonko, S. Kovalevskyi, V. Lishchenko, and F. Zots. Method for the detection of smallsized air objects by observational radars, Eastern-European Journal of Enterprise Technologies, № 2/9 (92), 2018, pp. 61-68.

DOI: https://doi.org/10.15587/1729-4061.2018.126509.

20. H. Khudov, S. Kovalevskyi, A. Irkha, V. Lishchenko, O. Serdiuk and F. Zots, The Proposals for Synchronization Positions of MIMO Radar System on the Basis of Surveillance Radars, in Intern. Scient.-Pract. Conf. Problems of Infocommunications. Science and Technology (PIC S\&T), 2019, pp. 547-551. DOI:

https://doi.org/10.1109/PICST47496.2019.9061284.

21. H. Khudov, I. Khizhnyak, V. Koval, V. Maliuha, A. Zvonko, V. Yunda, V. Nagachevskyi, and V. Berezanskyi The Efficiency Estimation Method of Joint Search and Detection of Objects for Surveillance Technical Systems, International Journal of Emerging Trends in Engineering Research, Vol. 8. № 3, 2020, pp. 813-819.

DOI: https://doi.org/10.30534/ijeter/2020/34832020.

22. I. Ruban, H. Khudov, V. Lishchenko, A. Zvonko, S. Glukhov, I. Khizhnyak, V. Maliuha, Y. Polonskyi, R. Kushpeta, The Calculating Effectiveness Increasing of Detecting Air Objects by Combining Surveillance Radars into The Coherent System, International Journal of Emerging Trends in Engineering Research, Vol. 8., № 4, 2020, pp. 1295-1301.

DOI: https://doi.org/10.30534/ijeter/2020/58842020.

23. H. Khudov, I. Khizhnyak, I. Yuzova, O. Baranik, G. Semiv, S. Bondarenko, and O. Tytarenko. The Optimization Technique for Joint Discrete Search and Detection of Observation Objects, International Journal of Emerging Trends in Engineering Research, № 8(2), 2020, pp. 533-538.

DOI: https://doi.org/10.30534/ijeter/2020/42822020.

24. V. Lishchenko, V. Chaliy, H. Khudov, and A. Zvonko. Proposals for Improving of Air Surveillance Informativity in MIMO Radar Systems Based on Two-Dimensional Radars, in Intern. Scient.-Pract. Conf. Problems of Infocommunications. Science and Technology (PIC S\&T), 2018, pp. 153-156.

DOI: https://doi.org/10.1109/infocommst.2018.8632052.

25. H. Khudov, A. Zvonko, I. Khizhnyak, V. Shulezko, V. Khlopiachyi, V. Chepurnyi, and I. Yuzova. The Synthesis of the Optimal Decision Rule for Detecting an Object in a Joint Search and Detection of Objects by the Criterion of Maximum Likelihood, International Journal of Emerging Trends in Engineering Research, № 8(2), 2020, pp. 520-524.

DOI: https://doi.org/10.30534/ijeter/2020/40822020.

26. V. Lishchenko, H. Khudov, B. Lisogorsky, O. Baranik, D. Holovniak, and O. Serdjuk The MIMO System on Based Existing Mechanical Rotation Radars with Wide Surveillance Area, in 2020 IEEE 40th International Conference on Electronics and Nanotechnology (ELNANO), 2020. P. 625-628. DOI:https://doi.org/10.1109/ELNANO.50318.2020.90887463 